\title{
Disappearance of Hemifacial Spasm After Ventriculoperitoneal Shunting in a Patient With Achondroplasia
}

\author{
-Case Report-
}

\author{
Shiro Yamashita, Yoshihito Matsumoto, Takashi TamiYa, \\ Masahiko KAWANISHI, Daisuke OGAWA, and Seigo NAGAO
}

Department of Neurological Surgery, Kagawa University School of Medicine, Kagawa

\begin{abstract}
A 15-year-old boy with achondroplasia developed right hemifacial spasm associated with headache, vomiting, and hearing disturbance. Computed tomography showed hydrocephalus. A ventriculoperitoneal shunt was placed. His hydrocephalus subsequently resolved, the hemifacial spasm and headache disappeared, and his hearing disturbance improved. The episodes of hemifacial spasm were probably related to a small posterior cranial fossa volume, the so-called crowding of the posterior fossa. Increased intracranial pressure due to hydrocephalus apparently contributed to further reduction in the posterior cranial fossa volume and led to the hemifacial spasms. In addition, his hearing disturbance may have been the result of dysfunction of the cochlear nerve due to the increase in intracranial pressure caused by hydrocephalus.
\end{abstract}

Key words: achondroplasia, hemifacial spasm, crowding of the posterior fossa, hydrocephalus, ventriculoperitoneal shunt

\section{Introduction}

Hemifacial spasm is caused by compression of the origin of the facial nerve, the so-called root exit zone. Vascular compression is the most frequent cause of hemifacial spasm, although various other structures may be involved in compression of this zone, such as arteries, veins, tumors, aneurysms, arteriovenous malformations, and thickened arachnoid membrane. ${ }^{8)}$ Microvascular decompression is widely used to treat hemifacial spasm. ${ }^{4)}$ Congenital abnormalities of the skull or crowding of the posterior fossa due to malformation can also lead to vascular compression. $2,6,7,10,11)$ Hemifacial spasm may occur if the apparent size of the posterior fossa is decreased by hemorrhage. ${ }^{3)}$

Achondroplasia is a short-limb dwarfism due to abnormal formation of the endochondral bone. Inheritance is autosomal dominant, but only $80 \%$ of cases manifest as mutations. Achondroplasia is often associated with stenosis of the foramen magnum due to occipital bone thickness and stenotic compression of the craniocervical junction. ${ }^{12)}$ Achondroplasia may be associated with crowding of the posterior fossa due to basilar impression ${ }^{6)}$ or thick occipital bone. ${ }^{12)}$ Achondroplasia is rarely associated with hydrocephalus caused by disorders in the cerebrospinal fluid (CSF) circulation due to basilar impression. ${ }^{6)}$ Hydrocephalus may cause hearing disturbance..$^{1,5,8,9)}$

We treated a patient with achondroplasia complicated with crowding of the posterior fossa and obstructive hydrocephalus manifesting as hemifacial spasm and hearing disturbance. Ventriculoperitoneal shunting resolved the hydrocephalus, and the episodic hemifacial spasms disappeared and his hearing disturbance improved.

Received March 1, 2004; Accepted August 23, 2004

Author's present address: S. Yamashita, M.D., Department of Neurosurgery, University of Michigan, Ann Arbor, Michigan, U.S.A. 


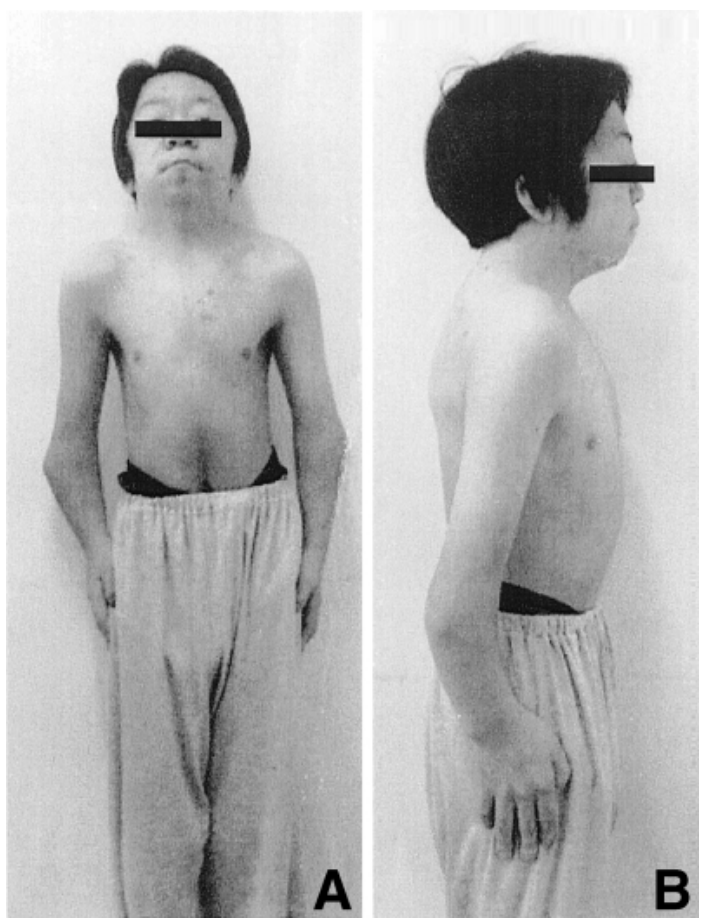

Fig. 1 Photographs of the patient at first presentation (A: anterior view, B: lateral view) showing the physical features of cartilaginous bone disorders such as four short limbs, short stature, and brachycephaly.

\section{Case Report}

A 15-year-old male visited our hospital in April 1998 complaining of spasms in the right eyelid. Achondroplasia was identified in early childhood and he exhibited the unique physical features of cartilaginous bone disorders such as four short limbs, short stature, and brachycephaly (Fig. 1). Initially he was treated with $400 \mathrm{mg}$ valproate daily at the Department of Pediatrics, but this medication was discontinued due to nausea. The spasm in the right eyelid spread to the right lip region in August. He reported frequent episodes of headache, vomiting, and right hemifacial spasm. Computed tomography revealed hydrocephalus and basilar impression (Fig. 2A, B). He was admitted to our department on October 26.

He continued to suffer headache and vomiting. Auditory testing revealed the presence of bilateral hearing disturbances (Fig. 3A). Radiography of the head revealed basilar impression, platybasia, and diastasis of the sagittal, coronal, and lambdoid sutures (Fig. 4). Magnetic resonance (MR) imaging demonstrated hydrocephalus and crowding of the posterior fossa associated with the basilar impres-
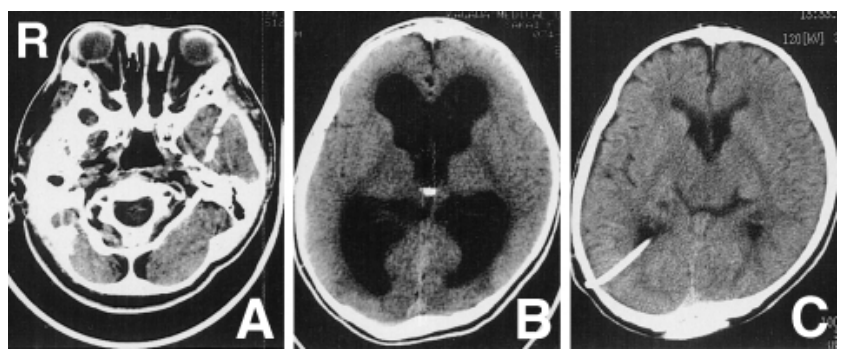

Fig. 2 A, B: Computed tomography (CT) scans at admission revealing basilar impression (A) and hydrocephalus (B). C: Postoperative CT scan showing disappearance of the hydrocephalus and a drainage tube from the posterior horn of the right lateral ventricle.

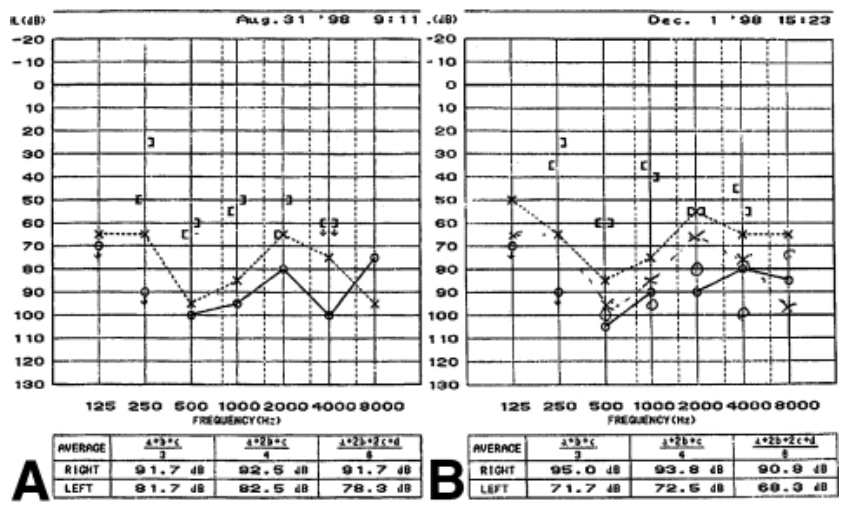

Fig. 3 A: Preoperative audiogram taken on August 31 showing bilateral combined hearing loss (right ear, 92.5 dB; left ear, $82.5 \mathrm{~dB}$ ). B: Postoperative audiogram taken on December 1 showing improvement of hearing by 10 dB in the left ear (right ear, $93.8 \mathrm{~dB}$; left ear, $72.5 \mathrm{~dB}$ ).

sion (Fig. 5A). We postulated that his headache and vomiting were symptomatic manifestations of increased intracranial pressure due to hydrocephalus.

A ventriculoperitoneal shunt was placed from the posterior horn of the right lateral ventricle using a pressure-programmable valve on November 17 . His initial intracranial pressure was $20 \mathrm{cmH}_{2} \mathrm{O}$ at the time of surgical intervention. The shunt valve pressure was set to $10 \mathrm{cmH}_{2} \mathrm{O}$ during the immediate postoperative period and later adjusted in response to changes in ventricle size and other symptoms such as head heaviness. Eventually, his hydrocephalus and hemifacial spasm disappeared at a valve pressure of $15 \mathrm{cmH}_{2} \mathrm{O}$ (Fig. 2C). His hearing disturbance improved (Fig. $3 \mathrm{~B}$ ), and the headache 

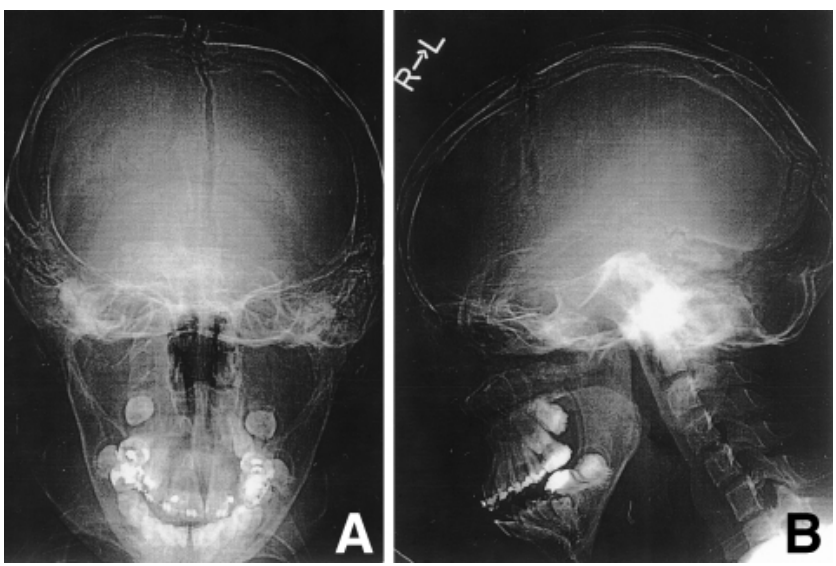

Fig. 4 Radiographs of the skull at admission (A: anteroposterior view, B: lateral view) showing diastasis of the sagittal, coronal, and lambdoid sutures, platybasia, and basilar impression.
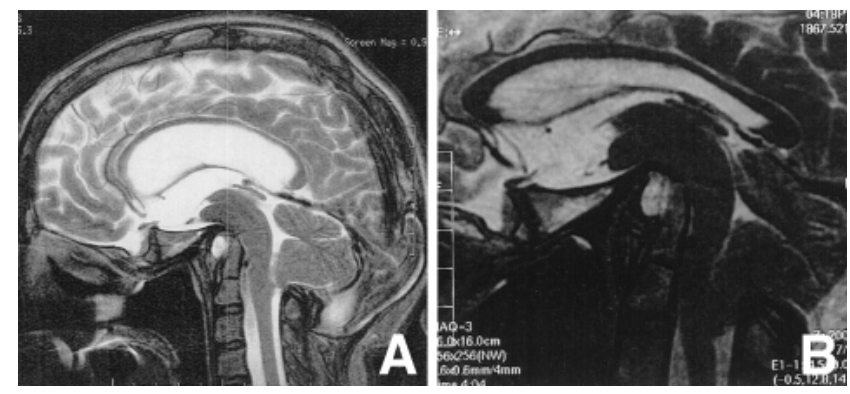

Fig. 5 A: Magnetic resonance (MR) image at admission showing hydrocephalus, aqueductal enlargement, and crowding of the posterior fossa due to basilar impression. No vessel was visualized near the exit zone of the right facial nerve. B: Follow-up MR image in February 2002 showing no recurrence of hydrocephalus. Note the normalization of the aqueductal size and of the deviation of the cerebellum and medulla oblongata in the downward direction.

and vomiting disappeared. His manifestations resolved and he was discharged on December 10. At the time of writing, there has been no recurrence of hydrocephalus (Fig. 5B), hemifacial spasm episodes, or hearing disturbance.

\section{Discussion}

Our patient had basilar impression associated with hydrocephalus and crowding of the posterior fossa. The crowding of the posterior fossa was exacerbated by the increased intracranial pressure due to hydrocephalus. He did not undergo MR cisternography, but MR imaging revealed no distinct mass lesion near the facial nerve, indicating that the episodes of hemifacial spasm were of vascular or arachnoid origin.

Ventriculoperitoneal shunting to decrease our patient's intracranial pressure resulted in disappearance of the hemifacial spasm and improvement of the hearing disturbance. No other surgical procedures were undertaken. Presumably the shunt caused changes in the CSF dynamics, and mitigation of posterior fossa crowding by decompression of the supratentorial space resulted in the abatement of the hemifacial spasm. Changes in CSF dynamics produced by spinal drainage have led to the disappearance of hemifacial spasm. ${ }^{8)}$ Hemifacial spasm after cerebellar hemorrhage associated with a cerebellar hematoma disappeared after removal and CSF drainage. ${ }^{3)}$ These previous cases and our case suggest that pulsatile flow of the CSF is involved in vascular or arachnoid compression of the facial nerve, resulting in hemifacial spasm, and that the changes in CSF volume and flow induced by continuous CSF drainage affect the compression site.

The most recent preoperative audiogram was obtained in August. Although he complained of further hearing loss, no other audiograms were taken until after surgery in November. Postoperative evaluation of his hearing indicated bilateral, albeit unequal, improvement based on the August test. Further audiograms taken between August and the date of operation may have demonstrated greater improvement. We postulate that the hydrocephalus and consequent increased intracranial pressure were factors in the exacerbation of his hearing loss. Various hypotheses regarding the etiology of hearing disturbance in patients with hydrocephalus have been presented. ${ }^{1,5,9,10)}$ The inner ear contains two ducts, the ductus perilymphaticus (DPL) and the ductus endolymphaticus (DEL), that are connected to the intracranial region. The DPL forms a connection between the scala tympani of the cochlea and the subarachnoid space in the posterior cranial fossa to facilitate passage of perilymphatic fluid and CSF. The DEL extends from the endolymphatic sac and connects with the subdural space. Increases in intracranial pressure may lead to an increase in inner ear pressure via these two ducts, especially the DPL, resulting in cochlear dysfunction and hearing disturbance.

In our patient, hearing improvement was greater in the left ear than the right. The caliber of the DPL and DEL shows marked individual variations and the effect of increased intracranial pressure on the 
inner ear is smaller if the duct caliber is small.5) Based on these considerations, we suggest that the dissimilar improvement in the right and left ears of our patient is attributable to differences in the caliber of the DPL and DEL.

The present patient with achondroplasia had hemifacial spasm and hearing disturbance which both improved after treatment for hydrocephalus without direct decompression of structures surrounding the facial and auditory nerves.

\section{References}

1) Barlas O, Gokay H, Turantan MI, Baserer N: Adult aqueductal stenosis presenting with fluctuating hearing loss and vertigo. J Neurosurg 59: 703-705, 1983

2) Dandy WE: Concerning the cause of trigeminal neuralgia. Am J Surg 24: 447-455, 1934

3) Hirano A, Ochi S, Kanno K: [A case of hemifacial spasm associated with a cerebellar hematoma]. No Shinkei Geka 22: 1159-1161, 1994 (Jpn, with Eng abstract)

4) Janetta PJ, Abasy M, Maroon JC, Albin MS: Etiology and definitive microsurgical treatment of hemifacial spasm: Operative techniques and results in 47 patients. J Neurosurg 47: 321-328, 1977

5) Kuwahara T, Muraki M, Kitamura S, Tuchiya N, Ninchoji T, Uemura K: [A case of hydrocephalus with hypacusis due to hemangioblastoma]. No Shinkei Geka 19: 385-389, 1991 (Jpn, with Eng abstract)
6) Luyendijk W, Matricali B, Thomeer RT: Basilar impression in an achondroplasic draft: causative role in tetraparesis. Acta Neurochir (Wien) 41: 243-253, 1978

7) Niijima $\mathrm{KH}$, Kondo A, Ishikawa J, Kim C, Itoh K: Familial osteodysplasia associated with trigeminal neuralgia. Case report. Neurosurgery 15: 562-565, 1984

8) Niijima KH, Yonekawa Y, Kaku Y: [Disappearance of hemifacial spasm following spinal drainage: A case report]. No Shinkei Geka 18: 577-580, 1990 (Jpn, with Eng abstract)

9) Saxena RK, Tandon PN, Sinha A, Kacker SK: Auditory functions in raised intracranial pressure. Acta Otolaryngol 68: 402-410, 1969

10) Tandon PN, Sinha A, Kacker SK, Saxena RK, Singh $\mathrm{K}$ : Auditory function in raised intracranial pressure. J Neurol Sci 18: 455-467, 1973

11) Yamamoto $Y$, Kondo A, Hanakita J, Nishihara K, Kinuta Y, Nakatani H: [Measurement and clinical significance of the posterior cranial fossa volume of patients with hemifacial spasm]. No Shinkei Geka 15: 243-248, 1987 (Jpn, with Eng abstract)

12) Yano S, Seki T, Hida K, Iwasaki Y: [Achondroplasia with respiratory disturbance: case report]. No Shinkei Geka 30: 51-55, 2002 (Jpn, with Eng abstract)

Address reprint requests to: S. Yamashita, M.D., Department of Neurosurgery, University of Michigan, 5550 Kresge I, Ann Arbor, MI 48109-0532, U.S.A. 